Revue Interventions économiques

Papers in Political Economy

$51 \mid 2014$

L'économie politique du "care". Entre continuité et changement

\title{
Une qualité d'emploi mitigée : qu'est-ce qui motive les aides à domicile?
}

A Mixed Quality of Employment: What Motivates Home Care Workers?

Ilda Ilse llama, Sophia Belghiti-Mahut et Alain Briole

\section{CpenEdition}

Journals

Édition électronique

URL : http://journals.openedition.org/interventionseconomiques/2134

DOI : 10.4000/interventionseconomiques.2134

ISBN : 1710-7377

ISSN : $1710-7377$

Éditeur

Association d'Économie Politique

\section{Référence électronique}

Ilda Ilse Ilama, Sophia Belghiti-Mahut et Alain Briole, « Une qualité d'emploi mitigée : qu'est-ce qui motive les aides à domicile? », Revue Interventions économiques [En ligne], 51 | 2014, mis en ligne le 01 novembre 2014, consulté le 10 décembre 2020. URL : http://journals.openedition.org/

interventionseconomiques/2134; DOI : https://doi.org/10.4000/interventionseconomiques.2134

Ce document a été généré automatiquement le 10 décembre 2020.

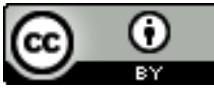

Les contenus de la revue Interventions économiques sont mis à disposition selon les termes de la

Licence Creative Commons Attribution 4.0 International. 


\title{
Une qualité d'emploi mitigée : qu'est-ce qui motive les aides à domicile?
}

\author{
A Mixed Quality of Employment: What Motivates Home Care Workers?
}

Ilda Ilse Ilama, Sophia Belghiti-Mahut et Alain Briole

\section{Introduction}

1 Compte tenu du vieillissement de la population dans nombre de pays, le secteur des relations de service est devenu l'un des axes majeurs de développement des économies contemporaines. Dans cet ensemble, les activités de service à la personne et plus particulièrement à la personne fragile connaissent la même dynamique, avec des particularités caractérisées par d'évidentes mutations sociétales ${ }^{1}$.

2 Le vieillissement de la population, l'évolution des formes de solidarité sociale et son inscription dans les politiques macro-économiques (Boyer, 2006), le passage d'une logique globale de contrôle à une logique de régulation des populations (Foucauld, 2004), pour ne citer que ces traits concourent à l'essor de ce secteur dont témoignent tant les prévisions en matière de besoins que l'évaluation des créations d'emploi.

3 L'arrivée récente d'opérateurs privés, du secteur assurantiel ou plus largement des services à la personne montre que cette activité longtemps dominée par l'action sociale est désormais prise dans un processus de rationalisation économique. Simultanément à l'enjeu de la création de valeur (et d'emplois) et de sa maîtrise, se pose aussi celui de la performance sociale qui ne peut se réduire au seul jeu des agents économiques engagés dans ce nouveau marché (Paugam, 2007). Le service d'aide à la personne reste porteur de valeurs caractéristiques du vivre ensemble (solidarité, relations intergénérationnelles...) que nombre d'observateurs estiment menacées dans la dynamique actuelle de croissance du secteur. 
4 La rationalisation économique et technique du secteur fait très rapidement surgir la question de la gestion de l'emploi, et plus précisément de la gestion des conditions d'emploi, entendue ici comme l'ensemble des relations contractuelles intégrant les conditions de travail, dans toutes leurs dimensions. C'est ainsi qu'on peut interpréter la question de la professionnalisation, qui va concerner la structuration tant du management que l'activité elle-même.

5 La professionnalisation est la plupart du temps envisagée surtout en référence directe à l'activité et aux compétences des salariés assurant le Service à la Personne. Elle est pourtant déterminée, dans une large mesure par la professionnalisation du management des organisations opérant dans ce secteur, de leurs capacités, notamment, à mettre en œuvre des outils de gestion adaptés à leurs finalités, à développer de véritable politique $\mathrm{RH}^{2}$.

6 Si certains s'en remettent à la rationalité immanente du marché, il est à peu près certain que dans cette perspective, l'activité du service à la personne se déploiera au moindre coût, pour une performance sociale largement discutable. Si d'un côté, on essaie de réguler ce secteur à partir d'une segmentation des acteurs (entreprises, autoentrepreneurs, associations...), il n'est pas certain que le résultat soit très différent. Suite à notre recherche, il nous semble que l'orientation d'où pourrait émerger une solution efficace est celle qui s'appuierait précisément sur la gestion des ressources humaines dans le secteur. L'amélioration de la qualité de l'emploi peut fournir le vecteur de déploiement de cette solution globale, plus positive pour les salariés du secteur et permettant d'assurer, de ce fait, un meilleur service à la personne.

7 Si on prend en considération cette dimension, essentielle à nos yeux, du développement économique à venir, la qualité de service apparaît comme un moyen particulièrement pertinent pour articuler les deux dimensions, sociale et économique, qui orientent la problématique de l'évolution du secteur. Qu'on la considère d'un point de vue méthodologique et technique, comme vecteur de transformation organisationnelle (Amadieu \& Cadin, 1996; Courpasson \& Livian, 1991), ou encore comme axe de développement d'une nouvelle forme d'échange (Gadrey, 1996), la qualité de service intègre un espace sur lequel peuvent se projeter l'ensemble des enjeux: la qualité de l'emploi.

8 On veut privilégier ici deux traits structurels parmi les plus significatifs de cette évolution: le déploiement d'une problématique de la performance dans un champ largement orienté par d'autres paradigmes des échanges sociaux, surdéterminée par l'arrivée de ces nouveaux acteurs que sont les entreprises, et les mutations des relations d'emploi qui s'y articulent nécessairement. Si le service à la personne reste encore pris dans des problématiques traditionnelles, des grilles d'analyse qui privilégient plus ou moins le niveau axiologique (Justice Vs Care, etc.), on peut, sans nier l'enjeu à ce niveau, au contraire conclure qu'il n'est pas naif d'envisager que son déploiement aura un impact considérable sur l'organisation sociale, à l'échelle d'une décennie, qui en modifie la nature comme le statut.

9 La gestion des ressources humaines peut être une dimension structurante de ce type d'activité, et la qualité de l'emploi le vecteur support de ce processus.Pour évaluer la qualité de l'emploi, la satisfaction au travail représente l'indicateur synthétique le plus adéquat (Vercernik, 2003). Le faible niveau des rémunérations, l'existence d'horaires le plus souvent flexibles et fractionnés, le temps partiel, subi plutôt que choisi et l'isolement 
des salariés sont des caractéristiques récurrentes de ce type d'emploi, désormais reconnues par la profession (Cerc ${ }^{3}$, 2008). Ces caractéristiques illustrent les difficultés que peuvent rencontrer les aides à domicile dans leur emploi, ce que confirment plusieurs études empiriques. Devetter et al., (2009) constatent ainsi que « la qualité globale des emplois est très médiocre ».

En se basant sur les problématiques liées à la qualité de l'emploi, cet article attire l'attention sur le fait que peu de travaux ont abordé la question de la motivation dans le secteur des services à la personne, et plus précisément celui de l'aide à domicile. L'étude réalisée au sein de deux associations dans la ville de Montpellier a pour but d'explorer la question suivante: alors que la qualité des emplois est assez mauvaise, comment expliquer que les aides à domicile se déclarent plutôt satisfaites de leur emploi et motivées par leur travail?

11 Notre préoccupation de recherche est de voir si au-delà des conditions de travail que connaissent les aides à domicile il y aurait des sources de motivation non exprimées qui leur permettrait de continuer à effectuer leur travail et qui pourraient contribuer à alimenter l'amélioration des emplois et du service en général.

Répondre à ces questionnements revient à présenter l'article en trois points. Nous présentons d'abord le concept de la qualité de l'emploi; puis quelques approches théoriques de la motivation au travail; et finalement les résultats, qui révèlent une appréciation plus nuancée de la part des salariés.

\section{Une qualité de l'emploi « objective » mauvaise}

13 Si la qualité de service s'impose à juste titre comme le dispositif majeur de régulation de ce secteur, la qualité de l'emploi pourrait constituer le support d'une alternative au néo taylorisme qui semble accompagner souvent la rationalisation du secteur ${ }^{4}$, à la fois une grille d'analyse et d'action.

\section{La qualité de l'emploi : un objet multidimensionnel et source de nombreuses investigations}

14 La notion de qualité de l'emploi ne saurait se limiter aux conditions de travail; elle renvoie aux caractéristiques de l'emploi et aux conditions extrinsèques dans lequel il se déroule (rémunération, vacances, heures de travail, horaire de travail, etc.) (Cloutier et al. , 2011 ; Cloutier, 2008). Le sens de la qualité de l'emploi est parfois limité par les données disponibles ainsi que les perspectives de recherche retenues (Krahn, 1992; Drolet et Morissette, 1998 ; Meisenheimer II, 1998; Hughes ; Green, 2006 ; CEE ${ }^{5}, 2006$; European Commission, 20083).

15 Mis en œuvre initialement en sciences économiques, le concept de la qualité de l'emploi ne cesse de faire l'objet de nombreuses recherches. Le positionnement selon lequel, le salaire constituerait l'unique déterminant de la qualité de l'emploi est discuté. L'utilisation d'un indicateur composite permettant d'exprimer de manière globale la qualité de l'emploiest plus appropriée (Rosenthal, 1989). Mc Dermid et al. (2001), mettent en place une conceptualisation plus complexe composée de quarante-trois facteurs regroupés en cinq dimensions : Conditions de travail Complexité du travail, Rémunération et sécurité, Avantages sociaux, Soutien de l'environnement de travail. Gupta et al. (1992) se 
positionnent pour leur part, sur douze déterminants : Charge horaire, Difficultés, Intérêt pour le travail, Cadre physique, Opportunités de promotion, Qualité de l'assistance, Opportunité de spécialisation, Sécurité de l'emploi, Rémunération, Avantages sociaux, Qualité des relations avec le supérieur, Qualité des relations avec les collègues. Si on envisage de mesurer la qualité des emplois, une première démarche est de questionner directement les individus sur leur satisfaction à travailler ${ }^{6}$ comme le soulignent Fremigacci et L'Horty (2005). C'est à partir de l'aspect satisfaction au travail que Handel (2005) étudie la progression de la qualité de l'emploi et du travail aux États-Unis pendant la décennie quatre-vingt-dix. Pour cela, l'auteur prend en considération la perception qu'ont les travailleurs des dimensions suivantes: rémunération, stabilité, heures de travail, conditions physiques, progression de carrière, travail enrichissant, autonomie professionnelle, relations employés-gestionnaire et relation entre les employés.

La Gestion des Ressources Humaines (GRH) quant à elle, s'est toujours inspirée des autres disciplines pour se développer (Yedder et Peretti, 2009).Savall et Zardet (2000) soutiennent que la création par la GRH de l'homme au travail est inspirée des courants de pensée en économie. En GRH l'objectif des rares travaux qui, selon la littérature, se sont imprégnés des questionnements en rapport avec la qualité, a été d'en délimiter les déterminants (Hunter 2000; Champion-Hugues, 2001). Ce positionnement est adéquat pour plusieurs raisons. Il favorise la prise en compte des attentes (Yedder et Peretti, 2009) d'une population quant à la qualité espérée d'un emploi de façon globale, en incorporant des dimensions variées. Cloutier et al., (2011) ont centré une de leur recherche sur les mères, en tentant de voir si elles étaient encore perdantes aujourd'hui dans la qualité d'emploi en dépit d'un rehaussement important de leur scolarité.

terifié comme un concept multidimensionnel, la Commission Européenne l'ayant définie à travers dix dimensions à caractères quantitatifs (Commission Européenne, 2001 ; Davoine et al., 2008). Sous cet ensemble, la qualité de l'emploi devient une notion plus large que la qualité du travail (job quality, work quality) qui est quant à elle centrée sur les conditions de travail.

Cloutier (2010) a particulièrement étudié la qualité de l'emploi chez les femmes au Québec, la comparant à la situation des hommes. La croissance de la féminisation de l'emploi est commune à une majorité des pays de l'OCDE (ILO, 2007; Johnston, 2005). Cependant, si les femmes sont présentes sur le marché du travail aussi bien que les hommes, elles occupent encore des emplois moins rémunérés, le temps partiel est récurrent et parfois involontaire, les emplois sont souvent de courte durée et le fait d'occuper des emplois peu ou pas qualifiés ne les aide pas à mettre à profit tous leur savoir, savoir-être, savoir-faire (Cranford et al., 2003 ; Lemière \& Marc 2006 ; Hughes et al., 2003). Les travaux de Cloutier, Bernard et Tremblay(2011) montrent toutefois que la qualité de l'emploi des femmes s'est améliorée au Québec au cours des deux dernières décennies (1997-2007).

Cette problématique de la qualité de l'emploi des femmes est tout à fait pertinente dans le secteur de l'aide à domicile. En effet, près de $98 \%$ des emplois sont occupés par des femmes dans ce secteur (Jany-Catrice, 2010); d'où la question centrale des facteurs de leur motivation au travail, que nous abordons ici, après une contextualisation de cette qualité de l'emploi dans le secteur de l'aide à domicile. 


\section{La qualité de l'emploi dans le secteur de l'aide à domicile : une réalité assez complexe}

20 manière significative dans les prochaines années (Bressé, 2003). En France, les plus de 60
ans, qui constituaient 11,5 millions de personnes en 1995, dépasseraient les 22 millions en
2050. Quant aux plus de 75 ans, sur la même période, ils passeraient de 3,5 à 11 millions (Gallouj, 2008).

D'après un sondage Opinionway pour l'observatoire de l'intérêt général (Auzanneau et Chardron, 2012), 90 \% des Français désirent vieillir chez eux (Auzanneau et Chardon, 2012 ; Franco, 2010). Le vivre chez soi est alors une liberté désirée de façon massive par les aînés. Cette liberté contient cependant des risques, et la responsabilité des autorités publiques revient à faire en sorte que cela se réalise dans de bonnes conditions (Franco, 2010). Les résultats de l'enquête $\mathrm{HID}^{7}$ montrent qu'un peu plus du quart des personnes âgées de 60 ans et plus ont une aide à leur domicile suite à un handicap ou un problème de santé (Dutheil, 2001). Cette aide a la possibilité d'être dispensée par trois types d'aidants. D'une part les aidants informels, qui représentent l'entourage des personnes et leur proposent une aide gratuite. Les aidants familiaux, qui sont des proches du bénéficiaire et en contrepartie sont rémunérés pour lui dispenser une aide, par des allocations comme l'Allocation Personnalisée d'Autonomie. Puis les aidants professionnels, qui sont rémunérés pour dispenser cette aide dans le cadre de leur métier. Toutefois, la qualité de ce dernier type d'aidant constitue une question pertinente à approcher dans ce contexte de pénurie, de crise et de tension de la profession.

est constaté que «la qualité globale des emplois est très médiocre » dans ce secteur (Dussuet, 2007). On y trouve des activités où les bas salaires sont répandus, plus de $70 \%$ des aides à domicile et des employés de maison ont de très bas salaires (Ponthieux et Concialdi, 2000), mais également une sécurité de l'emploi en trompe l'oeil (Maruani, 2006 ; Angeloff, 2000). Les durées de travail partiel se cumulent avec le faible niveau des qualifications reconnu. La première source d'insécurité économique est relative à la durée du travail, dans le champ des S.A.P ${ }^{8}$. Le temps partiel constitue la norme ${ }^{9}$, selon l'Enquête Emploi 67 \% des salariés « aides à domicile » et « employés de maison » exercent à temps partiel» (Dussuet, 2007; Doniol- Shaw et al., 2007). Les travailleurs à temps partiel involontaire représentent des "travailleurs contraints»: les employés qui occupent ce genre de poste sont confrontés à des grandes obligations dans la mesure où ils ne parviennent pas effectuer le nombre d'heures de travail souhaité (Cloutier et al., 2011). Des conditions de travail qui cumulent les pénibilités industrielles et celles du tertiaire (Volkoff et Gollac, 2007), viennent soutenir l'hypothèse de la médiocrité des emplois dans ce secteur (Devetter et al., 2009). Une pénibilité spécifique vient corroborer ces contraintes, à savoir celle de la mobilité forcée entre les interventions dans des domiciles, et pour des bénéficiaires différents (Jany-Catrice, 2007).

Parmi les autres pénibilités, Juhel (1998) met en avant l'exténuation physique et morale, les contacts avec les bénéficiaires qui peuvent être pesants, la monotonie des échanges, le manque de temps permettant d'effectuer toutes les tâches. Aussi le travail des aides à domicile comme celui des postiers ou des caissières de supermarché, contient diverses tâches de manutention. Elles doivent porter les courses, notamment les packs d'eau, transporter l'aspirateur d'une pièce à l'autre et même d'un étage à un autre, transporter

Revue Interventions économiques, 51 | 2014 
le linge dans le but de l'étendre, sortir les poubelles, déplacer le lit voire une commode pour nettoyer derrière. Les charges lourdes à porter ne se limitent pas uniquement aux tâches ménagères, car elles doivent aussi effectuer un "travail sur matériau humain " (Goffman, 1961), c'est- à-dire un travail physique réalisé sur les individus. Elles aident les personnes à sortir de leur baignoire, elles les déplacent de leur lit vers le fauteuil le matin et de leur fauteuil vers le lit le soir (Avril, 2006). Dans certains départements, les prestations des salariés se bornent au quart d'heure nécessaire au portage d'un repas, à la toilette, etc., cela représente plusieurs aller-retour pour l'employé qui subit la charge invisible de mobilité causée par le fractionnement de l'activité (Jany-Catrice, 2010). La signification commune tend à voir le travail effectué par les aides à domicile comme une simple prolongation de la domesticité, alors qu'il est d'une toute autre nature, complexité, capacité d'adaptation des salariés, autonomie ou variété des responsabilités (Dussuet, 2005). Aussi, de manière générale, ces salariés souffrent d'une absence de reconnaissance (Honneth, 2002 ; Margalit, 1996).

Ces quelques éléments sur la qualité de l'emploi des aides à domicile nous ont incités à nous intéresser à certaines approches théoriques de la motivation au travail. L'objectif est d'appréhender les aspects théoriques qui peuvent motiver les personnes dans leur travail pour tenter de comprendre, outre l'aspect financier, ce qui attache ces aides à domicile à leur travail décrit comme plutôt dénudé d'attrait. Comme nous l'avons indiqué, ceci pourrait constituer un vecteur d'amélioration de la qualité de l'emploi comme du service à la personne.

\section{Quelques approches de motivation au travail}

Il existe plusieurs définitions de la notion «motivation ». Toutefois, leur point commun c'est de définir la motivation comme la totalité des forces qui poussent une personne à avoir un comportement indiqué (Dolan et al., 2007).

La définition de Vallerand et Thill (1993) est souvent reprise dans les ouvrages de gestion des ressources humaines. «Le concept de motivation représente le construit hypothétique utilisé, afin de décrire les forces internes et/ou externe produisant le déclenchement, la direction, l'intensité et persistance du comportement" (Vallerand et Thill ; 1993). Kanfer (1990) rassemble des théories de la motivation dans un paradigme se rapportant aux besoins, mobiles et valeurs de l'individu. Ces théories mettent l'accent sur l'être humain par sa personnalité, ses dispositions, ses traits de caractère stables et ses valeurs, qui sont à l'origine de son comportement.

On peut distinguer d'une part les théories dites de contenu (Campbell et al., 1970) et les théories de processus d'autre part.

\section{Les théories de contenu : les facteurs qui incitent un individu à agir d'une certaine manière}

Les théories de contenu, comme le nom l'indique, s'intéressent au contenu de la motivation c'est-à-dire à l'ensemble des facteurs qui incitent l'individu à agir de telle ou telle façon. Elles tentent de répondre à la question «qu'est-ce qui motive les gens à travailler? » (Rojot et Bergmann, 1989), pour déterminer ces facteurs qui poussent les individus à agir. Dans ces théories s'intègrent quatre approches. 

catégories de besoins (physiologiques, de sécurité, sociaux, d'estime, d'actualisation) ordonnés suivant une structure organique. D'après Maslow, il est impossible que ces besoins soient ressentis au même moment, leur perception se fait de manière successive, et selon un ordre précis. Ensuite la théorie ESC d'Alderfer $(1969,1972)$, qui précise que les besoins sont directement joints à la motivation. Toutefois, ses investigations ne l'aide pas à élaborer une hiérarchie des besoins comme celle de Maslow (Dolan et al., 2007 ; Dolan et al., 1995). Alderfer $(1969,1972)$ organise les besoins en trois groupes, à savoir : les besoins d'existence (E), les besoins de sociabilité (S) et les besoins de croissance (C), d'où la théorie ESC.

Puis la théorie des deux facteurs de Herzberg. Selon Herzberg et al., (1959), certains facteurs peuvent procurer de la satisfaction et d'autres de l'insatisfaction. Les facteurs qui participent à la satisfaction ont un lien avec le travail lui-même et avec le sentiment de plénitude qui provient de cette satisfaction. Ces facteurs intrinsèques, nommés « facteurs de motivation" ou «facteurs moteurs", sont associés au contenu du travail ; ils se composent de: la réussite, la considération, l'autonomie, les responsabilités et l'avancement (Dolan et al., 2007 ; Dolan et al., 1995). En opposition, d'autres facteurs participent à affaiblir les attitudes négatives au travail sans pour autant causer un rendement important et constant de la part des salariés. Ces facteurs extrinsèques, nommés «facteurs d'hygiène» ou »facteur d'ambiance», ont un lien avec l'environnement de travail; ils se composent des politiques organisationnelles, la supervision (aspect technique), la politique salariale, les relations interpersonnelles, les conditions de travail et la sécurité de l'emploi (Dolan et al., 2007 ; Dolan et al., 1995).

Tout compte fait, d'après la théorie de Herzberg, il existerait chez les individus deux classes de besoins. Premièrement, tout comme dans le règne animal, l'être humain a besoin de se protéger du danger, de la privation et la douleur; deuxièmement, appartenant à la catégorie humaine, il éprouve le besoin d'être heureux et de se réaliser (Dolan et al., 2007).

Il y a des ressemblances entre les théories de Maslow, d'Alderfer et de Herzberg. Il est possible de comparer les besoins extrinsèques (facteurs d'hygiène) de Herzberg aux besoins physiologiques et de sécurité de Maslow ainsi qu'aux besoins d'existence et de solidarité d'Alderfer. Les besoins intrinsèques tels que Herzberg les définit (facteur de motivation) correspondent de façon simple aux besoins d'estime et d'actualisation de Maslow de même qu'aux besoins de croissance d'Alderfer (Dolan et al., 2007). Il y a toutefois une différence importante entre les trois théories. Celles de Maslow et Alderfer maintiennent que ce qui ne constitue pas une insatisfaction est incontestablement satisfaisant, et inversement. Ainsi, si la rémunération est bonne, on est satisfait, dans le cas contraire on est insatisfait. Herzberg ne partage pas cette logique, selon lui, il existe une division claire entre l'insatisfaction et la satisfaction. Alors, d'après la théorie des deux facteurs, les classifications de besoins s'élargissent sur des ensembles distincts les uns des autres. Les éléments de contexte de travail ou de contenu du travail ont des conséquences variées sur la satisfaction. Les premiers ont la possibilité de diminuer l'insatisfaction, tandis que les seconds uniquement peuvent engendrer une grande satisfaction au travail (Dolan et al., 2007).

Enfin, la théorie McCelland (1971), qui est connue pour ses recherches sur les besoins placés au sommet de la pyramide de Maslow. Cependant, cet auteur ne met pas en place une hiérarchie formelle des besoins. En revanche, il s'est concentré sur trois besoins qui

Revue Interventions économiques, 51 | 2014 
ont un lien certain avec l'environnement de travail : le besoin de réalisation, le besoin d'affiliation, et le besoin de pouvoir. Selon cette théorie toute personne ressent une dépendance soutenue envers l'un ou l'autre de ces besoins. Cependant, selon les événements, elle peut aussi éprouver les deux autres besoins, mais dans une faible mesure. La force d'un besoin et l'attitude qui s'en suit est variable selon les situations. Plus l'intensité d'un besoin est forte, plus la personne adopte des attitudes qui pourraient le satisfaire.

Outre les théories de contenu, on trouve aussi les théories de processus.

\section{Les théories de processus : les facteurs situationnels et les liens existants entre les besoins et les divers objets de l'environnement.}

Selon ces théories, les facteurs qui motivent un individu dans un contexte précis peuvent ne pas être adaptés pour un autre individu ou dans un autre contexte. Sans occulter l'importance des besoins et autres forces internes, les théories de processus, se concentrent sur les facteurs situationnels et au lien qu'il y a entre les besoins et les différents éléments du contexte. Ces théories se basent plus sur la direction et la durée d'une attitude que sur l'apparition de la motivation (Dolan et al., 2007).

Quatre théories de processus sont présentées ici. La première est celle des attentes. D'après la théorie des attentes, ou théorie de l'expectative, mis en œuvre par Vroom (1964), l'attitude d'une personne s'explique par la valeur qu'elle perçoit de ses conséquences. Cette théorie suggère aussi que la personne effectue des choix de manière consciente et raisonnée des moyens qui l'aideront à atteindre ses objectifs, afin que les efforts personnels ne soient pas fournis de façon coutumière, mais d'après une vision stratégique. Alors la personne réfléchit et évalue les différents choix, ce qui la conduit à prendre une décision basée sur des informations qui ont un lien avec la situation dans laquelle elle se situe. Cette théorie se distingue des diverses théories de contenu par l'intérêt qu'elle accorde aux choix rationnels des attitudes pouvant causer certaines conséquences (Dolan et al., 2007).

La deuxième est la théorie béhavioriste, employée principalement pour donner du sens au phénomène de l'apprentissage, elle peut aussi expliquer le phénomène de motivation au travail. Son positionnement est plus ou moins semblable à la théorie de Vroom, l'attitude dépend des conséquences qu'elle cause. Cependant au lieu de considérer l'attitude comme l'élément d'un choix personnel et rationnel, comme le fait Vroom, on l'examinera comme un élément des conditionnements (Dolan et al., 2007). Quatre techniques ont la possibilité d'être employées pour changer une attitude: le renforcement positif, le renforcement négatif, la punition et l'extinction.

La troisième est la théorie de l'équité de Adams $(1963,1965)$, elle soutient que les personnes sont encouragées par les injustices qu'elles voient quand elles comparent leurs liens intrants-extrants à celui d'autres individus ou groupes. Divers choix se présentent aux salariés qui voudraient rétablir l'équité : changer les extrants; changer les intrants ; changer le référent de comparaison; diminuer l'iniquité de manière cognitive ; changer d'emploi.

Quatrièmement la théorie des objectifs de Locke (1968; Locke et Latham, 1990), les personnes auront un rendement plus élevé dans la mesure où les objectifs qu'elles admettent d'atteindre sont laborieux, mais réalistes. Ainsi, pour qu'une personne soit 
motivée, elle doit avoir des objectifs qui sont clairs et précis, ils doivent être ardus, mais réalistes et la personne doit les accepter.

En somme, les théories de processus essaient d'expliquer la manière dont les forces interfèrent avec l'environnement afin de conduire la personne à avoir une attitude singulière (Dolan et al., 2007)

41 Par ailleurs, March et Simon (1974) soutiennent qu'il y a des distinctions nécessaires à opérer entre deux types de décisions prises par les employés. Face à son emploi, l'employé choisit: de quitter l'organisation, ou de rester dans l'organisation et produire ou encore rester dans l'organisation et ne pas produire. En rapport avec la question des raisons pour lesquelles les employés restent dans leur emploi, March et Simon (1974) précisent que «la motivation à la production émane d'un état présent ou prévu de mécontentement et de la perception d'un enchaînement direct entre la production individuelle et un nouveau stade de satisfaction ». Pour ces auteurs, les éléments qui encouragent le salarié à rester dans l'organisation et de produire ou à se conformer aux exigences de la hiérarchie de l'organisation se construisent certainement dans une théorie de l'influence. March et Simon (1974), précisent que des études empiriques des motivations individuelles de rester dans l'organisation et participer à son développement ont tendance à identifier : des facteurs relatifs aux objectifs des individus; des facteurs en relation avec les prévisions des conséquences et les facteurs relatifs à la totalité des choix tels qu'ils sont perçus au moment de la décision.

42 Même si la perspective s'en trouve élargie, la question de la motivation reste prise dans le paradigme de l'individualisme méthodologique et de ses références issues de la philosophie libérale qui considère la satisfaction comme exclusivement déterminée par la réalisation de besoins ou d'aspirations individuels, égoïstes.

Or chez Smith (1776) lui-même, le rapport à autrui s'intègre dans la réalisation du projet individuel, social par nature et de ce fait altruiste l'individu accorde du prix au bonheur de son prochain (Rojot, 2002). On peut voir là un point d'articulation possible de la théorie de la motivation à la théorie du « care » (Laugier et Paperman, 2004), par où pourrait se dépasser la rationalité purement instrumentale qui impacte sous une autre forme l'activité du service à la personne : privilégiant logiquement la technicité des emplois, elle occulte, voire stigmatise la dimension relationnelle des activités de l'aide à domicile, contribuant ainsi à leur dévalorisation. En effet, dans ce secteur il n'y a pas que le travail physique qui devrait être mis en avant, il y a d'autres compétences cachées qui devraient permettre davantage une valorisation de cette activité.

44 Face aux conditions de travail des aides à domicile décrites dans les quelques études mobilisées, ainsi que les éléments théoriques sur la question de la motivation, on peut s'interroger sur la représentation qu'en ont réellement ces aides à domicile, de même que sur les facteurs de leur motivation.

Les résultats d'une étude qualitative menée sur cette thématique vont tenter d'apporter quelques éléments de réponse dans la section qui suit. 


\section{Résultats de l'étude}

\section{Le recueil des données et l'option d'analyse retenue}

\section{Échantillon et technique de recueil d'information}

Cette étude s'est déroulée auprès de deux associations de l'aide à domicile à Montpellier. La première association (A) fait partie d'un grand groupement d'associations mutualisées d'économie sociale à Montpellier. La seconde association (B) est une association nationale, la majorité des salariés interrogés sont prestataires. Considérés comme étant un des modes d'entretien le plus employé en gestion (Roussel et Wacheux, 2005), à l'aide d'un guide d'entretien, des entretiens individuels semi-directifs d'une durée moyenne de 1 heure ont été effectués, auprès de 18 aides à domicile, 2 agents de service technique (AST). L'échantillon (le détail en annexe 1) est composé principalement de femmes et l'âge moyen des interviewés est de 38,5 ans pour les aides à domicile, 33,5 ans pour les AST.

\begin{tabular}{|c|c|c|c|c|c|c|}
\hline Fonction & Age & Sexe & Diplôme & $\begin{array}{l}\text { Type } \\
\text { contrat }\end{array}$ & $\begin{array}{l}\text { Temps } \\
\text { de } \\
\text { travail }\end{array}$ & $\begin{array}{l}\text { Nbre } \\
\text { Heure/mois }\end{array}$ \\
\hline $\begin{array}{l}\text { Agent à } \\
\text { domicile }\end{array}$ & 27 & $\mathrm{~F}$ & Master 2 rech. en $\mathrm{SG}^{12}$ & $\begin{array}{l}\text { CDD - } \\
\text { Etudiante } \\
\text { remplaçante }\end{array}$ & $\begin{array}{l}\text { Temps } \\
\text { partiel }\end{array}$ & $\begin{array}{l}108 \text { heures } \\
\text {-Mandataire }\end{array}$ \\
\hline $\begin{array}{l}\text { Auxiliaire } \\
\text { de vie }\end{array}$ & 40 & $\mathrm{~F}$ & DEAVS & CDI & $\begin{array}{l}\text { Temps } \\
\text { complet }\end{array}$ & $\begin{array}{l}140-156 \text { heures } \\
\text { En fonction des } \\
\text { remplacements }\end{array}$ \\
\hline $\begin{array}{l}\text { Auxiliaire } \\
\text { de vie }\end{array}$ & 40 & $\mathrm{~F}$ & DEAVS & CDI & $\begin{array}{l}\text { Temps } \\
\text { partiel }\end{array}$ & 160 heures \\
\hline $\begin{array}{l}\text { Auxiliaire } \\
\text { de vie }\end{array}$ & 59 & $\mathrm{~F}$ & DEAVS & CDI & $\begin{array}{l}\text { Temps } \\
\text { partiel }\end{array}$ & 120 heures \\
\hline $\begin{array}{l}\text { Auxiliaire } \\
\text { de vie }\end{array}$ & 21 & $\mathrm{~F}$ & DEAVS + Aide-soignante & CDI & $\begin{array}{l}\text { Temps } \\
\text { partiel }\end{array}$ & 72 heures \\
\hline $\begin{array}{l}\text { Agent à̀ } \\
\text { domicile }\end{array}$ & 48 & $\mathrm{~F}$ & $\begin{array}{l}\text {-Secrétariat de direction } \\
\text {-Sans diplôme dans le secteur }\end{array}$ & CDI & $\begin{array}{l}\text { Temps } \\
\text { partiel }\end{array}$ & 32 heures \\
\hline $\begin{array}{l}\text { Agent à } \\
\text { domicile }\end{array}$ & 32 & $\mathrm{~F}$ & $\begin{array}{l}\text { Master } 2 \text { professionnel - sans } \\
\text { diplome Dans le secteur }\end{array}$ & CDI & $\begin{array}{l}\text { Temps } \\
\text { partiel }\end{array}$ & 70 heures \\
\hline $\begin{array}{l}\text { Employé à } \\
\text { domicile }\end{array}$ & 35 & $\mathrm{~F}$ & $\begin{array}{l}\text { BEP carrière sanitaire et } \\
\text { sociale }\end{array}$ & CDI & $\begin{array}{l}\text { Temps } \\
\text { partiel }\end{array}$ & 120 heures \\
\hline $\begin{array}{l}\text { Agent à } \\
\text { domicile }\end{array}$ & 40 & $\mathrm{~F}$ & $\begin{array}{l}\text { BEP couture (sans diplôme } \\
\text { dans le secteur) }\end{array}$ & $\mathrm{CDD}$ & $\begin{array}{l}\text { Temps } \\
\text { partiel }\end{array}$ & 52 heures \\
\hline $\begin{array}{l}\text { Agent à } \\
\text { domicile }\end{array}$ & 30 & $\mathrm{~F}$ & $\begin{array}{l}\text { Master } 2 \text { Gestion des } \\
\text { Ressources Humaines (sans } \\
\text { diplôme dans le secteur) }\end{array}$ & CDI & $\begin{array}{l}\text { Temps } \\
\text { partiel }\end{array}$ & 32 heures \\
\hline $\begin{array}{l}\text { Agent à } \\
\text { domicile }\end{array}$ & 37 & $\mathrm{~F}$ & $\begin{array}{l}\text { Deug en Tourisme (sans } \\
\text { diplôme dans le secteur) }\end{array}$ & CDI & $\begin{array}{l}\text { Temps } \\
\text { complet }\end{array}$ & 135 heures \\
\hline $\begin{array}{l}\text { Auxiliaire } \\
\text { de vie }\end{array}$ & 47 & M & DEAVS & CDI & $\begin{array}{l}\text { Temps } \\
\text { partiel }\end{array}$ & $\begin{array}{l}150 \text { heures en } \\
\text { fonction des } \\
\text { remplacements }\end{array}$ \\
\hline $\begin{array}{l}\text { Auxiliaire } \\
\text { de vie }\end{array}$ & 40 & $\mathrm{~F}$ & DEAVS & CDI & $\begin{array}{l}\text { Temps } \\
\text { partiel }\end{array}$ & 130 heures \\
\hline $\begin{array}{l}\text { Auxiliaire } \\
\text { de vie }\end{array}$ & 34 & $\mathrm{~F}$ & DEAVS & CDI & $\begin{array}{l}\text { Temps } \\
\text { partiel }\end{array}$ & 135 heures \\
\hline $\begin{array}{l}\text { Auxiliaire } \\
\text { de vie }\end{array}$ & 45 & $\mathrm{~F}$ & $\begin{array}{l}\text { - Bac Professionnel } \\
\text {-Secrétariat } \\
\text {-Comptabilité } \\
\text {-DEAVS }\end{array}$ & CDI & $\begin{array}{l}\text { Temps } \\
\text { partiel }\end{array}$ & 144 heures \\
\hline $\begin{array}{l}\text { Agent à } \\
\text { domicile }\end{array}$ & 40 & $\mathrm{~F}$ & $\begin{array}{l}\text { Animatrice (sans diplôme } \\
\text { dans le secteur) }\end{array}$ & $\begin{array}{l}\text { CDD- } \\
\text { remplaçante }\end{array}$ & $\begin{array}{l}\text { Temps } \\
\text { partiel }\end{array}$ & 110 heures \\
\hline $\begin{array}{l}\text { Agent à } \\
\text { domicile }\end{array}$ & 21 & $\mathrm{~F}$ & Sans diplôme & CDI & $\begin{array}{l}\text { Temps } \\
\text { partiel }\end{array}$ & 149 heures \\
\hline $\begin{array}{l}\text { Agent à } \\
\text { domicile }\end{array}$ & 50 & $\mathrm{~F}$ & Diplôme de sténodactylo & CDI & $\begin{array}{l}\text { Temps } \\
\text { partiel }\end{array}$ & 135 heures \\
\hline $\begin{array}{l}\text { Agent à } \\
\text { domicile }\end{array}$ & 39 & $\mathrm{~F}$ & $\begin{array}{l}\text { Master } 2 \text { recherche en droit } \\
\text { privé (sans diplôme dans le } \\
\text { secteur) }\end{array}$ & CDI & $\begin{array}{l}\text { Temps } \\
\text { partiel }\end{array}$ & 85 heures \\
\hline $\begin{array}{l}\text { Agent à } \\
\text { domicile }\end{array}$ & 30 & $\mathrm{~F}$ & $\begin{array}{l}\text { Master } 2 \text { rechecrehe en droit } \\
\text { privé (sans diplôme dans le } \\
\text { secteur) }\end{array}$ & CDI & $\begin{array}{l}\text { Temps } \\
\text { partiel }\end{array}$ & 85 heures \\
\hline
\end{tabular}

\section{Option d'analyse retenue}

47 L'analyse de contenu (Bardin, 1993 ; Savall et Zardet, 2004) d'exploration a été retenue comme option d'analyse des discours des 20 personnes constituant l'échantillon. En effet, il s'agissait de dénombrer des éléments d'un contenu. On a cherché à établir une sorte d'inventaire des diverses opinions exprimées ou des arguments utilisés. Le texte a été découpé et étudié en fonction des idées ou des mots qu'il contenait. En d'autres termes nous avons recensé les principales phrases exprimées par les acteurs, appelées phrases témoins (Savall et Zardet, 2004). Cette procédure nous a permis de mettre en évidence la 
perception de la qualité de l'emploi des acteurs interrogés, ainsi que les différents facteurs de motivation.

Après avoir rappelé les conditions de travail et d'emploi, tout en soulignant et illustrant par des verbatim la relative satisfaction des salariées, nous discuterons deux propositions opposées : une motivation largement "intrinsèque " d'un côté et une "motivation » contrainte « faute de mieux » d'un autre côté.

\section{Les résultats : une perception de la qualité de l'emploi assez hétérogène.}

L'objectif de cette partie est de présenter les résultats relatifs à la perception de la qualité de l'emploi à travers ses dimensions limitées à celle de la rémunération, des conditions de travail, la sécurité de l'emploi et des relations avec l'employeur. En effet, il est question ici d'explorer les représentations des aides à domicile en matière de qualité de l'emploi concernant ces dimensions.

Si on envisage de mesurer la qualité des emplois, une première démarche est de questionner directement les individus sur leur satisfaction à travailler (Fremigacci et L'Horty, 2005). La satisfaction au travail représente l'indicateur synthétique le plus pertinent pour mesurer la qualité de l'emploi ${ }^{10}$ (Vecernik, 2003). Elle synthétise les informations que les enquêtes les plus classiques ne peuvent dévoiler (l'ambiance de travail par exemple) et prend compte des valeurs et des aspirations de chacun (Kalleberg et Vasey, 2005).

\section{La rémunération : entre l'absence de choix et la non-prise en compte de l'effort réalisé.}

51 Pour quelques aides à domicile la rémunération est satisfaisante, mais souvent cette satisfaction peut être confondue avec le manque de choix, car, l'une des raisons évoquées est celle de la conformité de la rémunération à la loi. Le manque d'expérience dans ce domaine peut justifier ce positionnement.

Interviewé $1:$ «je suppose qu'elle est conforme à la loi, le SMIC je crois il est à 8,33 et les nombres d'heures travaillées égales au nombre d'heures payées (...) je suis satisfaite de cette rémunération ». Interviewé 7: "J'aurai bien aimé gagner plus, mais vous savez que c'est le SMIC ».

En revanche nombreux sont les interviewés qui ont manifesté leur insatisfaction par rapport à la rémunération. Cette dernière ne compense pas l'effort effectué, l'énergie dépensée. Par ailleurs tous les paramètres psychologiques entourant le métier d'aide à domicile ne sont pas pris en compte dans la rémunération.

Interviewé 2: "Non, moi je trouve que c'est mal payé par rapport au travail et à l'énergie qu'on donne ». Interviewé 6 : «Sincèrement moi je ne suis pas satisfaite, je trouve que pour l'effort qu'on fait, on est très mal rémunéré ». Interviewé 8 : "Non. Je trouve que on n'est pas assez rémunéré pour le métier qu'on fait, $\mathrm{y}$ a l'aide à domicile y a pas que l'entretien, (...)»

53 La perception qu'ont nos interviewés de leur rémunération a-t-elle une incidence sur l'idée qu'ils se font de la sécurité de leur emploi? 


\section{La sécurité de l'emploi : une croissance des personnes âgées rassurante, mais un environnement de travail assez fragile.}

récurrentes sont : l'augmentation du nombre de personnes âgées, la pérennité de l'emploi dans le secteur depuis son origine, le fait d'avoir un contrat à durée indéterminée.

Interviewé 3 : «De plus en plus il y a des personnes âgées, les gens vont vivre beaucoup plus longtemps, donc je crois que oui il y a une sécurité de l'emploi (...)». Interviewé 11 : «(...) effectivement on est en CDI, c'est pas du tout un secteur qui est en danger, ça n'a rien à voir avec toutes les boîtes qui ferment ou qui ne ferment pas, il y a toujours de la demande ».

Tandis que d'autres salariés manifestent leur insatisfaction et précisent qu'il n'y a pas de sécurité d'emploi dans ce secteur. Les raisons qui reviennent sont: la fragilité des bénéficiaires de l'aide entrânant leur décès, le nombre insuffisant des heures de travail qui peuvent encore diminuer quand le bénéficiaire se fait hospitaliser.

Interviewé 1: "Non parce que c'est axé sur des gens malades et des personnes âgées qui ont une espérance de vie qui en général pas longue et du coup tu perds ton emploi ». Interviewé 10: «Mais il est aussi précaire, car les personnes âgées meurent et on est parfois amené à changer de personne sans pour autant avoir un nombre d'heures suffisant $»$.

La littérature mentionne des conditions de travail des aides à domicile peu attractives (Cerc, 2008) qu'en est-il de la perception de nos interviewés?

\section{Les conditions de travail : un environnement de travail parfois complexe}

Pour certaines aides à domicile, les conditions de travail sont satisfaisantes, car l'association leur remet le matériel de travail nécessaire.

Interviewé 1: «Vu que le matériel de sécurité de travail je veux dire les gants, les blouses ça nous est fourni par l'association donc sur le plan hygiénique ça va, oui je suis satisfaite ». Interviewé 4 : «Oui ça va quand même et puis ici si on a besoin de quelque chose on lui demande, là ou il $\mathrm{y}$ a la famille où je travaille ils ont tout le matériel ça va. Si on a besoin de quelque chose, il suffit de lui dire et là même si les enfants ne sont pas là ça va ».

Mais dans la mesure où l'intervention s'effectue à domicile les conditions de travail ne sont pas toujours satisfaisantes, car le statut social du bénéficiaire de l'aide, l'insalubrité du domicile peuvent les défavoriser. Pour d'autres aides à domicile l'insatisfaction est assez prononcée et n'hésitent pas à dénoncer certains dysfonctionnements : manque d'information précise sur la gestion des plannings, absence au niveau de l'encadrement. Un manque d'information sur les pathologies des bénéficiaires qui pourrait représenter un danger potentiel pour les intervenantes en cas de pathologies contagieuses a été souligné. Et même les intérimaires des maisons de retraite ne sont pas informées des pathologies des bénéficiaires. Le lieu de travail en lui-même constitue une difficulté. Le brouillage au niveau des interventions, car il y a une différence entre une aide ménagère et une auxiliaire de vie. L'aide ménagère n'a pas de diplôme, elle n'a peut être aucune formation ni information sur le déroulement des tâches dans le secteur, elle n'a jamais travaillé avec les personnes âgées et ne sait pas comment se comporter avec eux. 
L'auxiliaire de vie quant à elle, elle a reçu une formation, elle est peut être mieux aguerrie à certaines situations.

Interviewé 1 : «par contre ça dépend des personnes chez qui on va travailler autant tu trouveras des personnes qui sont très bordéliques chez elles et très sales autant tu trouveras des personnes, mais vraiment rangées même des personnes à la limite maniaque (...) ». (...) » Interviewé 2 : «L'association je trouve qu'elle est vraiment nulle on n'a pas de suivi (...) y a rien y a pas de lien. On n'a pas le sentiment d'être dans une structure encadrée ou quand on a un pépin on peut leur en parler (...) ». Entretien 11 : «on se retrouve chez les personnes qui ont des pathologies et on ne sait pas, donc on découvre sur le terrain, moi il y a des personnes aujourd'hui je sais qu'elles sont malades je soupçonne que ça soit Alzheimer, mais j'ai personne qui me l'a dit que cette personne-là est malade, a la maladie d'Alzheimer (...) ». Interviewé $\underline{8}$ : «Non, pastout le temps, mais dans l'ensemble ça va. C'est toujours difficile du fait qu'on a à faire à des gens, il faut gérer ». Interviewé 10 : « un jour en étant chez un bénéficiaire l'infirmière n'arrivant pas j'étais obligé de lui faire sa toilette intime sachant que je n'étais pas obligée de le faire, car c'était au-delà de mon intervention. Par ailleurs je m'occupe d'une autre personne elle n'aime pas être nettoyée par l'aide-soignant et donc c'est à moi de la nettoyer le soir au niveau de sa partie génitale qui nécessite des soins médicaux ».

Les aides à domicile de notre échantillon présentent des conditions de travail qui ne sont pas toujours satisfaisantes. Qu'en est-il des relations avec leur employeur?

\section{La relation avec l'employeur : une bonne relation, qui est parfois ternie par une absence de communication.}

De cette étude il ressort que le mode d'intervention n'a pas d'impact sur le degré de satisfaction du salarié, car il y a autant de satisfaits que d'insatisfaits dans les deux cas. Les salariés qui sont satisfaits le justifient à travers les bonnes relations entretenues avec l'employeur.

Interviewé 1 : «Très bonne relation, elle me satisfait. Au niveau de l'association je trouve que c'est organisé dans leur mode de fonctionnement et dans leur système de travail dans ce sens que y a des fiches, on est répertorié (...), on a le contact direct avec le chef de secteur on peut discuter ». Interviewé 7 : « Dans l'ensemble ça va».

61 En revanche, en matière d'insatisfaction l'argument le plus récurrent est encore le manque de communication, la mauvaise gestion des plannings comme nous l'avons souligné précédemment au niveau des conditions de travail. Cette insatisfaction au niveau des relations avec l'employeur n'est pas particulière aux aides à domicile, même les AST le sont. Bien qu'ils avancent que les relations existantes sont des relations de respect, mais il ressort aussi une insuffisance de communication.

Interviewé 2: «il y a beaucoup de choses qui ne me satisfont pas, on n'a pas quelqu'un en face de nous(...) ». Interviewé 5 : «très peu de relations donc tout se passe toujours par téléphone». Interviewer 19: «ce que je peux critiquer c'est le climat entre la direction et le personnel de travail, on est souvent mis sous pression, un travail sous pression ne garantit pas une bonne qualité de travail, une bonne qualité de tâche, de l'exécution de tâche ».

En somme qu'il s'agisse de la rémunération, de la sécurité de l'emploi, des conditions de travail, ou des relations avec l'employeur, on constate une hétérogénéité des points de vue. Ces différences de points de vue s'expliquent uniquement par la perception des interviewés. Nous rappelons que tous les déterminants de la qualité de l'emploi ne sont 
pas présents dans ce document. Au niveau des deux organisations, les perceptions des salariés se rejoignent, aucune particularité n'a été constatée.

Les éléments d'insatisfaction, en somme assez fréquents, interpellent et conduisent à s'interroger sur les facteurs qui motivent les aides à domicile. Notons que la qualité des services semble de façon assez certaine avoir un lien avec la qualité de l'emploi des salariés qui les réalisent. Il n'est pas évident de travailler avec efficacité et sérénité auprès de bénéficiaires fragiles quand on est soi-même en tant que salarié dans une posture de faiblesse économique et sociale. Le point qui suit permet de mettre en évidence les multiples facteurs de motivation des aides à domicile aussi intéressant soient-ils. En faisant référence aux théories de contenu (Campbell et al., 1970), la question suivante a été posée aux interviewés : qu'est-ce qui vous motive dans votre travail ? À l'aide des données recueillies deux grandes propositions sont mises en avant: une motivation intrinsèque liée à une forme de rationalité plus normative ou affective d'un côté et une «motivation" contrainte pour des salariées dont la faible employabilité peut les contraindre à accepter ces emplois « faute de mieux ».

\section{Des facteurs de motivation largement « intrinsèques » d'un côté et une motivation contrainte « faute de mieux » d'un autre côté.}

Deci et Ryan $(1985,2002)$ présentent la motivation intrinsèque où l'action est menée dans le but et le plaisir que la personne trouve à l'action, sans expectative de récompense externe. La motivation extrinsèque est celle où l'action est suscitée par un événement extérieur à la personne (punition, récompense, pression sociale obtention de l'approbation d'une personne tierce). En observant de plus près nos données, la motivation de nos interviewés est largement intrinsèque, dans le sens où ils ont du plaisir à faire leur travail (Herzberg et al., 1959) et n'attendent pas forcément un retour de la part des personnes aidées.

\section{La prédominance des facteurs de motivation intrinsèques.}

\section{Le « care » : une importante source de motivation}

L'ensemble des auteurs reconnaissent que la traduction du «care » en français n'est pas évidente. C'est en même temps un verbe qui signifierait "s'occuper de ", faire attention ", "prendre soin de ", et où la traduction française serait « soin ", "sollicitude ", " souci d'autrui ». (Paturel, 2010, p.183; Garrau et Le Goff, 2010, p.5). Chacune de ces interprétations renvoie pratiquement à un élément du «care » (Garrau et Le Goff, 2010) : La notion "d'attention » accentue sur une façon de voir le monde et les tiers; celles de «souci " ainsi que de «sollicitude» renvoient à la façon d'être angoissé par eux; puis celle de «soin ", à la façon de s'en occuper réellement. La notion de "care " se situe à l'intersection entre la disposition - une attention à l'autre qui se développe dans la conscience d'une responsabilité à son égard, d'un souci de son bien-être - et de l'activité l'ensemble des tâches individuelles et collectives visant à favoriser ce bien-être (Garrau et Le Goff, 2010, p. 5). Fisher et Tronto (1991, in Tronto 2009, p. 147) définissent le care comme « une activité générique qui comprend tout ce que nous faisons pour maintenir, perpétuer et réparer notre «monde », de sorte que nous puissions y vivre aussi bien que 
possible. Ce monde comprend nos corps, nous-mêmes et notre environnement, tous les éléments que nous cherchons à relier en un réseau complexe, en soutien à la vie ». Le " care » se développe dans la volonté de faire valoir l'attention aux autres «singuliers ", aux détails de la vie humaine enracinée dans l'expérience vécue (Paturel, 2010).

En rapport avec tous ces éléments, les interviewés disent que ce qui les motive c'est qu'ils aiment aider les personnes âgées. Leur attrait pour l'aide aux autres nous fait penser au " care ». La dimension culturelle est un élément à prendre en compte dans ces facteurs de motivation, car ce qui motive certains interviewés c'est de s'occuper des personnes âgées et le faire en pensant à leurs grand-parents : aider les personnes âgées c'est se souvenir des « anciens » et combler le manque lié à cette absence.

Interviewé $1:$ :C'est comme un peu une activité bénévole, bénévole dans le sens que tu fais quelque chose de bien pour quelqu'un». Interviewé 3 : «J'aime bien aider les personnes âgées 》. Interviewé 14 : «J'aime bien aider les personnes, aider les gens c'est s'aider sois même aussi ». Interviewé 18 : «Ce qui me motive c'est que j'ai l'occasion justement de m'occuper de quelqu'un, de malades, d'invalide, de gravement malade jusqu'au bout ». Entretien 19: «Ça me plait bien de m'occuper des personnes âgées parce que c'est des personnes nécessiteuses, vu leur âge, vu leur problème à l'âge qu'ils ont ".

Interviewé 1: "Dans ma culture, je viens de l'Afrique centrale, on est habitué à vivre en famille, j'ai toujours été habitué à être avec mes grands-parents. Ils me manquent et ça me motive d'être avec les personnes âgées, de m'occuper d'eux, car ils rappellent un peu les miens ». Interviewé 10: «Je n'ai pas eu l'occasion de connaître mes grands-parents donc ce travail me permet de me rapprocher des personnes âgées, c'est comme si je rattrapais le temps perdu ».

Dans leur discours, les interviewés précisent aussi être motivés par le contact humain, le relationnel, la gentillesse et la reconnaissance des personnes âgées. Ces éléments pourraient relever des facteurs de motivation intrinsèque, car les aides à domicile n'attendent forcément pas un retour de la part des bénéficiaires de l'aide.

\section{Le contact humain et le relationnel, la gentillesse, la reconnaissance des personnes âgées : d'importants facteurs de motivation intrinsèque.}

Les interviewés déclarent être motivés par le contact avec les personnes âgées, les relations qu'ils entretiennent entre elles. Ils sont aussi motivés par la communication et l'écoute accordée aux bénéficiaires. Le comportement et la culture des personnes âgées agissent de façon positive sur la motivation des aides à domicile et des AST.

Interviewé 1 : «(...) y a le contact humain et moi j'aime le contact humain». Interviewé 2 : "C'est le contact humain, c'est humain, (...) y a un service, et y a la qualité humaine et y a le contact humain ». Interviewé 3 : "C'est surtout tout ce contact qu'il y a entre moi et les personnes que j'aime Interviewé 15: «Ma motivation c'est de communiquer, avec les personnes âgées d'être à leur écoute " Interviewé 1: "y a les personnes âgées je trouve qu'elles sont intéressantes dans leur petite bulle elles sont très intéressantes elles te racontent les histoires d'avant, les techniques. Exemple un monsieur il me racontait comment on tapait le journal, l'encre qu'ils utilisaient, les personnes âgées ont toujours des histoires intéressantes (...) je préfère ce travail-là à un autre travail d'étudiant ». Interviewé $\underline{11}$ : « ha oui les moments que j'aime bien aussi avec les personnes âgées c'est quand elles commencent à parler, à raconter, (...), elles ont des connaissances sur Montpellier, Montpellier dans les années 20 c'était comme ça, dans les années 30 ». 


\section{Plaisir de travailler et sentiment d'utilité au travail}

69 Dans un de ses ouvrages, Thévenet (2004) énonce le plaisir de travailler des salariés. On retrouve ce même plaisir dans le discours des interviewés comme facteur de motivation au travail. Aussi ils se sentent utiles dans leur travail. En effet, l'homme au travail éprouve la nécessité de se sentir utile, car de façon naturelle il cherche à comprendre les raisons pour lesquelles il effectue son travail. Il affectionne participer à un ouvrage et se rendre compte de l'importance de son œuvre sur son environnement (Laurent, 2013). Nos interviewés précisent être motivés par le fait de leur utilité au travail. Le plaisir de travailler au sens de Thévenet (2004) vient également accentuer ce sentiment d'utilité au travail qu'ont les à domicile.

Interviewé 3 : «J'avoue que j'aime ça, (...)». Interviewé 12 : «Ma motivation c'est que je me sens bien dans mon travail, il m'épanouit ». Interviewé 14 : « vous savez quand on sort d'une journée et/ou on aide les gens on est fier, on se dit aujourd'hui on a réalisé quelque chose aujourd'hui dans notre vie » Interviewé 19: "c'est aussi quelque chose qui me plait parce que sinon je le ferais mal ». Interviewé 17 : «Moi ma motivation c'est pour moi-même c'est pour ma personne avant tout c'est quelque chose que je fais pour moi ».

Interviewé 5 : «(...) je me sens bien dans ce travail je me sens utile, j'ai l'impression d'être utile aux personnes ». Interviewé 11 : «se sentir utile (...) c'est ce côté même indispensable, (...). Je me sens indispensable, c'est un petit peu comme si je faisais du bénévolat sans être payé en fait, y a ce côté-là social ou je me retrouve aussi, (... )». Interviewé 12: "ma motivation c'est que quand même de me rendre utile d'avoir une utilité Interviewé 18 : «Ce qui me motive (...) et d'être utile voilà ». ».

\section{L'évolution de carrière et les finances}

70 Une autre orientation des interviewés est celle de faire carrière et d'évoluer dans le secteur de l'aide à domicile. Des interviewés ont précisé qu'ils envisagent d'évoluer, ne pas terminer leur carrière comme auxiliaire de vie ou bien aide ménagère, mais de devenir soit responsable de secteur ou bien aide-soignante.

\section{Une motivation contrainte « faute de mieux »}

71 Faute de mieux, ces salariés sont parfois obligés d'accepter cet emploi. En d'autres termes, il y en qui font ce travail par défaut, parce qu'ils n'ont pas eu le choix, d'où une motivation par défaut.

Interviewé 2 : «J'adore, je ne me vois pas faire autre chose (...) j'aimerais bien aller plus loin j'aimerai bien faire aide-soignante je n'aimerais pas rester auxiliaire de vie, j'aimerais faire aide-soignante, ça j'aimerais bien parce que je vois les aidessoignantes ce qu'elles font et j'aime ». Interviewé 8 : « (...) je souhaitais travailler dans le sanitaire, le sociale. En fait mon projet c'est de préparer le concours d'aidesoignant (...)». Interviewé 11: "J'ai envie d'accéder à un poste d'adjoint de secteur $»$.

Interviewé 6: «ma motivation c'est de subvenir à mes besoins, à mes besoins du quotidien ». Interviewé 7: "C'est l'aspect alimentaire qui me motive, c'est un boulot alimentaire ». Interviewé 11 : «Ma motivation bon déjà de travailler, d'avoir un salaire». Interviewé 16: "Moi tant que j'ai ça je travaille, je n'ai pas de préférence, je le fais pour gagner ma vie, tant que je travaille y a un salaire ».

Interviewé 3 : «Je suis venue, je n'ai pas trouvé dans mon secteur, j'ai commencé avec ça et je me retrouve encore toujours ici (...). J'ai été obligé de faire une formation puisque je me suis intéressée aux personnes âgées ». Interviewé 13 : « $\mathrm{Si}$ 
vous voulez demandez-moi toutes les nanas que je connais elles le font parce qu'elles n'ont pas le choix quoi. T'es obligé en quelque sorte. Après c'est obligé que ça devient... c'est un travail comme un autre, mais bon ce n'est pas un travail que tout le monde ou que la plupart des gens aimeraient en avoir quoi ».

En somme, les théories de contenu, en l'occurrence la théorie des deux facteurs de Herzberg et al., (1959) ont été d'un réel support pour cet article. Des mobiles de motivation ont été évoqués: le "care", "le contact humain et le relationnel, la gentillesse, connaissance des personnes âgées », «le plaisir de travailler, et le sentiment d'utilité au travail », de même que «l'évolution de carrière et les finances ». Au sens de Herzberg et al., (1959) ces facteurs ont un lien avec le sentiment de plénitude pour des salariées satisfaits. Mais faute de mieux, des salariés se trouvent obligés de faire ce travail. Les théories de processus ont une utilité, mais elles ne correspondaient pas à nos observations. La majorité des interviewés de notre échantillon reste dans leur travail parce qu'ils aiment ce qu'ils font et l'environnement de travail le permet également. Nos résultats permettent d'indiquer que la rationalité purement instrumentale est largement dépassée, dans le sens où les compétences cachées des aides à domicile se révèlent. Il n'y a pas que le ménage, mais aussi, l'amour du métier et l'aide apportée aux bénéficiaires.

\section{Conclusion}

Des recherches empiriques soutiennent que l'emploi dans ce secteur est médiocre (Devetter, Jany-Catrice, Ribault, 2009). Bien d'autres encore révèlent des insatisfactions des salariés sur certaines composantes de leur emploi (Contassot et Maurage, 2008; PES $34,2008)$. Les résultats de cette étude montrent qu'il s'agisse de la rémunération, de la sécurité de l'emploi, des conditions de travail, ou des relations avec l'employeur, on constate une hétérogénéité des points de vue.

En matière de rémunération certains salariés interviewés déclarent être satisfaits, car ils estiment que leur salaire est conforme à la loi c'est-à-dire au SMIC. Alors que d'autres sont insatisfaits et précisent que le salaire ne prend pas en compte l'effort effectué et l'énergie dépensée. Nos interviewés soutiennent aussi que les paramètres psychologiques qui entourent leur métier ne sont pas valorisés dans la rémunération. En matière de sécurité de l'emploi, le sentiment de certains salariés satisfaits est relié à la pérennité de la demande dans le secteur. Tandis que pour d'autres la fragilité et le décès des personnes dont ils s'occupent rend cette sécurité éphémère. Concernant les conditions de travail, certains interviewés évoquent une insuffisance au niveau de l'encadrement, de l'information sur les pathologies des bénéficiaires de l'aide ainsi que de la communication.

Bien qu'il y ait cette dualité au niveau de la perception de la qualité de l'emploi, les résultats sur les facteurs de motivation des aides à domicile aux personnes âgées sont intéressants. Une prédominance des facteurs de motivation intrinsèque a été constatée. Ici les aides à domicile font leur travail avec plaisir, parce qu'elles aiment le faire, elles aiment prendre soin des personnes aidées. En effet, les aides à domicile s'affirment motivées, et motivées surtout par le fait qu'elles aiment aider les personnes âgées et retrouvent une satisfaction et une certaine reconnaissance personnelle à le faire. Elles sont également sensibles aux relations entretenues avec les personnes âgées et les temps d'échanges partagés avec elles. Aussi la gentillesse des bénéficiaires de l'aide ne cesse d'attiser la motivation des aides à domiciles envers leur travail. En ce sens les aides à 
domicile se sentent utiles au travail et trouvent de ce fait leur travail stimulant et cela les motive davantage. L'évolution dans le secteur de l'aide à domicile vient corroborer cet inventaire des facteurs de motivation des aides à domicile. Très peu ont affirmé être motivé par l'aspect financier. personnes âgées sur la motivation des salariés et nous incite à approfondir le questionnement et donc la recherche sur cette dimension du « care » et ses impacts sur la motivation ou l'implication des salariés. Nous sommes d'ailleurs en train de poursuivre la réflexion dans le cadre d'une étude analogue sur les divers secteurs de services à domicile au Québec, afin de déterminer si les secteurs d'économie sociale et privés ou autres présentent les mêmes sources de motivation et d'engagement que ce que nous avons observé en France, ou si la situation est différente. Cela permet de poursuivre les travaux d'analyse de ce secteur d'emploi, largement féminin, et relativement peu étudié du point de vue de la qualité de l'emploi, comme des politiques et pratiques de la gestion de la main-d'œuvre.

\section{BIBLIOGRAPHIE}

Adams, J. Stacy (1963). « Toward an Understanding of Inequity ", Journal of Abnormal and Social Psychology, vol. 067, no 5, p. 422-436.

Adams, J Stacy (1965). Inequity in social Exchange", dans L Berkowitz (dir), Advance in Experimental Social Psychology, 2em éd., New York, Academic Press, p. 202-210.

Alderfer, Clayton .P. (1969). « An Empirical Test of a new Theory of Human Needs », Organizational Behavior and Human Performance, vol. 4, p. 142-175.

Alderfer, Clayton.P. (1972). Existence, Relatedness and Growth : Human Needs in Organizational Settings, New York, Free Press.

Amadieu, Jean-François et Cadin, Loïc. (1996). Compétence et organisation qualifiante, Economica. 112 pages.

Angeloff, Tania (2000), Le temps partiel, un marché de dupes ? Syros. 236 pages.

Auzanneau, Nadia. et Chardron, Stéphanie (2012). Dépendance et MAD / pour Observatoire de l'intérêt général. OpinionWay pour LJ CORP. Rapport mars, 16p.

Avril, Christelle (2006). « Le travail des aides à domicile pour personnes âgées : contraintes et savoir-faire ", Le Mouvement Social, 2006/3 no 216, p. 87-99. 
Bardin, Laurence (1993). L'analyse de contenu, PUF, Le psychologue.

Boyer, Luc (2006), Management des hommes : historiques, grands acteurs et autres, méthodes, outils perspectives, Organisation. 231 pages.

Bressé, Sophie (2003). « L'enjeux de professionnalisation du secteur de l'aide à domicile en faveur des personnes âgées ", Retraite et société, no39, p. 119-143.

Campbell, J. P., Dunnette, M. D., Lawler, E. E. Ill, \& Weick, K. E., Jr. (1970), Managerial behavior, performance, and effectiveness. New York: McGraw-Hill, 1970.

Conseil Emploi Revenus Cohésion Sociale (CERC) (2008). « Les services à la personne, rapport $\mathrm{n}$ - 8 ", janvier, La Documentation française, 147 pages.

Champion-Hugues, R. (2001). “Totally integrated employee benefits”. Public Personnel Mangement, 2001.

Cloutier, Luc (2008). La qualité de l'emploi au Québec, développements conceptuels et création d'une typologie. Etat actuel de la réflexion. Québec, Institut de la statistique du Québec. 47p.

Cloutier, Luc (2010). L'évolution de la qualité de l'emploi des femmes et des hommes au Québec entre 1997 et 2007 : l'ascenseur de la scolarisation et le fardeau des responsabilités familiales. Thèse de Doctorat. Université Montréal. Faculté des arts et des Sciences.

Cloutier, Luc., Bernard, Paul., et Tremblay, Diane-Gabrielle (2011). Activité professionnelle et responsabilités familiales : les mères sont-elles encore perdantes dans leur qualité de l'emploi ? Relations Industrielles, Volume 66, p. 256-278.

Cornet, Annie (2005). « Flexibilité du temps de travail : des stratégies différenciées pour les hommes et les femmes? ", in Matthieu de Nanteuil et Assâad El Akremi, La société flexible ERES «Sociologie économique », p. 291-309.

Cranford, C. J., L.F. Vosko, N. Zukewich (2003). « The Gender of Precarious Employment in Canada ", Relations industrielles/Industrial Relations, vol 58, no.3,p,454-480.

Courpasson, David., et Livian, Yves Frédéric (1991). «Le développement de la notion de compétence : glissement sémantique ou idéologie ? ", Revue française de Gestion des Ressources Humaines. No3, p3-10.

Commission Européenne., « Employment In Europe, Luxembourg : Commission européenne, Direction Générale de l'emploi, des affaires sociales et de l'égalité des chances, 2001.

Contassot, Philippe., et Maurage, Vincent (2008). S'engager pour la qualité de l'emploi d'aide à domicile. Aract/ Aide à domicile/ Pôle emploi service 34/Réunion débat/, juin, http :

www.languedoroussillon. aract.fr, 2008.

Davoine, Lucie., Erhel, Christelle., et Guergoat-Larivière, Mathilde (2008)."Monitoring quality in work : European Employment Strategy indicators and beyond", juin, International Labour Review, vol. 147 (2008), $\mathrm{N}^{\circ} 2-3$.

Davoine, Lucie (2007).La qualité de l'emploi : une perspective européenne, Thèse de Doctorat en Sciences Economique, Université Paris 1Panthéon-Sorbonne.

Devetter François-Xavier., Janice-Catrice, Florence et Ribaultv Thierry (2009). Les services à la personne, La Découverte.

Dolan Simon M., Gosselin Eric., et Carrière Jules (2007). Psychologie du travail et comportement organisationnel. Préface de Jean-Marie Peretti. E. Gaetan Morin. Chenelière Education. 494 pages. 
Dolan Simon M., Lamoureux Gérald., et Gosselin Eric. (1995). Psychologie du travail et des organisations. E. Gaetan morin. 500 pages.

Doniol-Shaw, Ghislaine., Lada, Emmanuelle., et Dussuet, Annie (2007). Les parcours professionnels des femmes dans les métiers de l'aide à la personne : leviers et freins à la qualification et à la promotion, Rapport de recherche LATTS, novembre, 276p.

Drolet, Marie et René Morissette (1998). Recent Canadian Evidence on Job Quality by Firm Size. Analytical Studies Branch - Research Paper Series, Statistics Canada, No. 11F0019MPE No. 128, Ottawa.

Dusset, Annie (2007). L'enjeu de la santé au travail dans les associations de services àdomicil elearning.londonmet.ac.uk/wlri/.../05 \%20Risque \%20et \%20sante/Dussuet.doc

Dussuet, Annie (2005). Travaux de femmes : enquêtes sur les services à domicile, L'Harmattan, Paris/ Budapest/Toronto, coll. « Le travail du social », 216p.

Dutheil, Nathalie (2001). Les aides et les aidants des personnes âgées. Etudes et Résultats. Drees, n - 142,.novembre,12p.Les aides et les aidants des personnes âgéeswww.sante.gouv.fr/IMG/pdf/ er142.pdf +1

Fisher, Berenice. et Tronto, Joan (1991). « Toward a feminist theory of care », in E. Abel et M. Nelson (dir.), Circles of Care : Work and Identity in Women's Lives, State University of New York, Albany, NY, 1991, 1991, p. 40.

Foucault, Michel (2004). Sécurité, Territoire, Population, Seuil- Gallimard, 373 pages.

Franco, Alain (2010). Rapport de la mission « vivre chez soi ». Présenté à Madame Nora Berra.Secrétaire d'Etat en charge des Aînés. travail-emploi.gouv.fr/IMG/pdf/

Rapport_Vivre_chez_soi.pdf

Fremigacci, Florent., L'Horty, Yannick (2005). « La qualité de l'emploi en France : Tendance et Cycle ", novembre, Centre d'étude de recherche, 2005. Novembre. $\mathrm{N}^{\circ} 51,37 \mathrm{p}$.

Gadrey, Jean (1996). L'Economie des services, La Découverte, « » Repères », 128 pages.

Gallou,j Camal (2008). L'économie des services à la personne, Wolters Kluwer, 211pages.

Garrau, Marie. et Le Goff, Alice (2010). Care, Justice et dépendance. Introduction aux théories du Care. Collection, Philosophies, Puf, 160 pages.

Goffman, Erving. (1961). Asiles, Etudes sur la condition sociale des malades mentaux, Paris, Editions de Minuit, 1968 (1961), p. 121-130.

Green, Francis (2006). Demanding work: The Paradox of Job Quality in the Affluent Economy. Princeton : Princeton University Press.

Gupta, Nina., Jenkins G.Douglas., et Beehr Terry.À (2005) “The effects of turn over on perceived job quality". Group and Organization Management, 2005.

Handel Michael-J., “Trends in Perceived Job Quality in the affluent economy”, février, Princeton University Press, Princeton, (2005). In Cloutier L., «La qualité de l'emploi au Québec, développements conceptuels et création d'une typologie. Etat de la réflexion, Québec ", Institut de la statistique du Québec. 2008.

Herzberg, Frederick., Mausner, Bernard et Snyderman, Barbara Bloch. (1959). The Motivation to Work, New York, John Wiley \& Sons.

Honneth, Axel (2002). La Lutte pour la reconnaissance, Cerf. 240p. 
Hughes, Karen, Lowe, Graham S., Schellenberg, Grant, (2003). “Men's and Women's Quality of work in the New Canadian Economy", Reseach Report, W/19, Work Network, February, Canadian Policy Reseach Network (CPRN), Ottawa, 68p.

Hunter, L-W (2000). "What deterrmines Job quality in nursing homes ?" Industrial \& Labor Relations Review, Vol 53., $n^{\circ}$ 3. p. 463-481.

International Labour Organisation (ILO), (2007). « Global Employment Trends for women, Brief », march, Genève" 20p.

Jany-Catrice, Florence. (2010). Les services à la personne : des emplois pas comme les autres. La documentation Française, p69-87.

Jany-Catrice, Florence (2007). « La société postindustrielle et les salariés de mobilité. Le cas des aides à domicile ", juin, colloque à l'institut ville en mouvement, 2007, 12p.. www.ville-enmouvement.com/.../la-societe-post-industrielles-et-les-salar...

Juhel, B (1998). L'aide ménagère et la personne âgée : petites et grandes manœuvres autour d'un espace de vie à partager, Paris-Montréal, L'Harmattan, 230 pages.

Johnston, Grant (2005). « Women's participation in the labour force », New Zealand Treasury, Working Paper 05/06, June, Wellington, 39p.

Kalleberg, Arn.L., et Vaisey, Stephen. (2005). « Pathways too good job : work quality among the machinist », British Journal of Industrial Relations, vol. 43, n 3, pp. 431-454.

Kanfer, Ruth (1990). "Motivation theory and industrial and organizational psychology", In M.D. Dunette et L.M. Hough (Eds), Handbook of industrial and organizational psychology, Palo Alto, CA : Consulting Psychologists Press, vol. 1. p. 75-170.

Krahn, Harvey (1992). Quality of Work in the Service Sector. General Social Survey Analysis Series, no. 6. Ottawa : Statistics Canada.

Lemière, Séverine., Marc, Céline, (2006). "Qualité des emplois et genre : les effets croisés des statuts et des horaires de travail”, Centre d'Économie de le Sorbonne, Paris, 29p.

Locke, Edwin A (1968). « Toward a Theory of Task Motivation and Incentives », Organizational Behavior and Human Performance, no3, p. 157-189.

Locke, Edwin A et Latham, G.P. (1990). A Theory of Goal Setting and Task Performance, Englewood Cliffs (N.J.), Prentice-Hall.

Maslow, Abraham.H. (1943). «A thery of Human Motivation », Psychological Review, vol. 50, no3, p. 370-396.

Maruani, Margaret (2006). Travail et emploi des femmes, la Découverte, 123 pages.

March, James.G. et Simon, Herbert (1974). Les Organisations. Traduit par Rouchy, J.C et Prunier G. Préface de Crozier M. Organisation et Sciences humaines. Collection dirigées par Filloux, J.C. Dunod.

McClelland, David. (1971). Assessing Human Motivation, New York, General Learning Press.

Mc Dermid S.M., Hertzog J.L., Kensinger K.B. et Zipp J.F. (2001). “The Role of Organization Size and Industry in job Quality and Work-Family Relationship", juin, Journal of Family and Economic Issues, Vol 22., $\mathrm{n}^{\circ}$ 2. p191- 216.

Meisenheimer II, Joseph R. 1998. «The Services Industry in the 'Good' versus ‘Bad' Jobs Debate ». Monthly Labor Review, 121 (2), 22-47. 
Paturel, Dominique. (2010). « Un exemple d'activité régulatoire et de ses difficultés » Témoignage d'une assistance de service social du travail (AS), Revue internationale de psychologies, 2010/39 Vol XVI, p. 173-188.

Paugam, Serge (2007), Repenser la solidarité, PUF.

Pôle emploi service Hérault., « Etude sur les conditions d'emploi de services à la personne », juin, www.pes34.com/files/file/.../Etude-Conditions-de-l--emploi-janvier-2008.pdf

Ponthieux Sophie. et Concialdi, Pierre (2000). « Bas salaire et travailleurs pauvres : une comparaison entre la France et les États-Unis », La Revue de l'Ires, 2000.

Rojot, Jacques. et Bergamann, Alexandre. (1989), Comportement et organisation. Paris, Vuibert Gestion, 396 pages.

Roussel, Patrice., Wacheux, Frédérick. (2005). Management des ressources humaines. Méthodes de recherche en sciences de gestion, De Boeck université, 114pages.

Rosenthal, N.H (1989). « More than wages at issue on job quality debate ». Monthly Labor Review, 1989.In Yedder M-B., Perretti J.M., « Qualité de l'emploi : Diversité internationale des attentes, septembre, Revue Management \& Avenir.2009.

Savall, Henri., et Zardet, Véronique (2000). « L'apport de l'Economie à la GRH », Personnel, 2000.

Savall, Henri et Zardet, Véronique (2004). « Recherche en Sciences de Gestion : Approche Qualimétrique ", Economica.

Thevenet, Maurice (2000), Le plaisir de travailler : favoriser l'implication des personnes, Paris : Ed. d'Organisation.

VecernikEC J., « Skating on Thin Ice : A Comparison of Work Values and Job Satisfaction in CEE and EU Countries ", International Journal of Comparative Sociology, 2003. InDavoine L. (2007), La qualité de l'emploi : une perspective européenne, Thèse de Doctorat en Sciences Economique, Université Paris 1Panthéon-Sorbonne.

Vallerand, Robert. J. et Thill, Edgar.E (1993). Introduction à la psychologie de la motivation. Coll. Vigot, Ed. De la Chenelière. Montréal.

Volkoff, Serge et Gollac, Michel (2007). Les conditions de travail, La découverte.

Vroom, Victor. H. (1964). Work and Motivation, New York, Wiley.

Yedder Moez-Ben., Perretti, Jean.Marie (2009). « Qualité de l'emploi : Diversité internationale des attentes, septembre, Revue Management \& Avenir.2009.

\section{NOTES}

1. Un indice certain de la centralité de cette question réside dans la structuration d'un secteur social dans les pays émergents (Travail social \& ESS, Séminaire International de Meknès, 10-11 mai 2010).

2. Ressources Humaines.

3. Conseil de l'emploi des revenus et de la cohésion sociale.

4. À bien des égards, le service à la personne pourrait s'identifier, dans sa gestion, au secteur du nettoyage.

5. Communauté Économique Européenne.

6. Et c'est cette démarche que nous avons adoptée au moment du recueil de nos données.

7. Handicap - Incapacités - Dépendances. 
8. Service à la personne.

9. Comme le souligne Cornet $(2005$, p. 4) « un tiers de l'emploi féminin est un emploi à temps partiel ».

10. "work satisfaction is the best indicator of job quality"

\section{RÉSUMÉS}

La qualité de l'emploi dans le secteur de l'aide à domicile pose un certain nombre de questionnements. Dans ce secteur les emplois sont d'une part pourvus avec difficulté et d'autre part marqués par des conditions de travail peu attractives, le secteur souffrant globalement d'une image plutôt négative. Cet article se propose à travers une étude qualitative de repérer d'abord ce qui peut différencier la satisfaction des salariés en matière de qualité de l'emploi ; ensuite les facteurs motivant leur travail, dans un environnement qui n'est pas toujours favorable.

Les résultats obtenus montrent que la perception de la qualité de l'emploi n'est pas homogène, mais produit des représentations plutôt convergentes, révélant des orientations problématiques en matière de gestion des ressources humaines. Une diversité de facteurs apparait en effet, motivant les aides à domicile dans leur travail parmi lesquels on retrouve le " contact humain ", les «relations avec les personnes» dont elles s'occupent, et toute une série d'éléments relationnels qui relèvent $d u$ « care ». Ceci permet d'envisager des politiques publiques améliorant à la fois la qualité de l'emploi et du service.

The employment quality in the sector of home care services raises a number of questions. In this sector, job positions are hard to fill and they meet unattractive working conditions. Overall, the sector is suffering from a rather negative image. This article, through a qualitative study, identifies the differentiation factors of employee satisfaction in terms of employment quality, and the work motivation factors, in an environment that is not always favorable.

The results show that the perception of employment quality is not homogeneous, but rather produces convergent representations revealing issues with human resources management. A variety of factors appear to motivate the home care services employees in their work. Among which are the "human touch", "relationships with people" in their care, and a variety of relational elements that fall within the "care". This can lead to the development of public policies supporting better quality of service as well as employment conditions.

\section{INDEX}

Mots-clés : aide à domicile, care, motivation, qualité de l'emploi, service à la personne

Keywords : care, home help, human services, motivation, work employment 